\title{
PREFERENCE INTENSITY IN MCDM WHEN AN ADDITIVE UTILITY FUNCTION REPRESENTS DM PREFERENCES
}

\author{
A. MATEOS, A. JIMENEZ, E.A. AGUAYO, P. SABIO \\ Departamento de Inteligencia Artificial, Universidad Politécnica de Madrid \\ (UPM), 28660 Boadilla del Monte, Madrid, Spain
}

\begin{abstract}
We propose a new method for ranking alternatives in multicriteria decision-making problems when there is imprecision concerning the alternative performances, component utility functions and weights. We assume decision maker's preferences are represented by an additive multiattribute utility function, in which weights can be modeled by independent normal variables, fuzzy numbers, value intervals or by an ordinal relation. The approaches are based on dominance measures or exploring the weight space in order to describe which ratings would make each alternative the preferred one. On the one hand, the approaches based on dominance measures compute the minimum utility difference among pairs of alternatives. Then, they compute a measure by which to rank the alternatives. On the other hand, the approaches based on exploring the weight space compute confidence factors describing the reliability of the analysis. These methods are compared using Monte Carlo simulation.
\end{abstract}

\section{Introduction}

In multicriteria decision-making, the classical additive multiattribute utility model is considered to be a valid approach in many practical situations ${ }^{2}$. Incorporating imprecision concerning weights and/or component utilities is one way of extending the model to closely describe real situations: less information is usually available than is needed to determine the best alternative ${ }^{9}$. Sarabando and Dias ${ }^{7}$ give a brief overview of approaches within the multiattribute utility theory framework to deal with incomplete information.

A recent approach is to use information about each alternative's intensity dominance, known as dominance measuring methods. Ahn and Park' were the first to propose a dominance measuring method. This method computes both dominating and dominated measures and then derives a net dominance. Net dominance is used as a measure of the strength of preference in the sense that a greater net value is better. Mateos et al. ${ }^{5,6}$ propose and compare two alternative methods aimed at improving Ahn and Park's methods. Mateos et al. ${ }^{5}$ consider uniformly distributed intervals to model imprecision concerning weights, whereas Mateos et al. ${ }^{6}$ consider ordinal relations among attribute weights. 
A second approach to deal with imprecision is the Stochastic Multicriteria Acceptability Analysis (SMAA) ${ }^{3}$. SMAA computes acceptability indices, which measure the variety of different preferences that rank each alternative as best. This information can be used to classify the alternatives as more or less acceptable and as unacceptable. However, SMAA ignores information about the other ranks. This problem is solved in Lahdelma and Salminen ${ }^{4}$ using the SMAA-2 method, which extends the analysis to the sets of weight vectors for any rank from best to worst for each decision alternative.

In this paper, we extend and improve the methods proposed in Mateos et al. $^{5,6}$. Instead of weight intervals for each attribute or ordinal relations among attribute weights, we assume weights can follow independent normal distributions or fuzzy numbers (triangular or trapezoidal). In Section 2 we introduce the extension of dominance measuring methods proposed in Mateos et al. ${ }^{5,6}$. Section 3 reports a simulation study of the methods outlined in previous sections. Finally, some conclusions are considered.

\section{A New Preference Intensity Method (NPIM)}

We consider a set of alternatives $A_{1}, \ldots, A_{m}$ evaluated on attributes $X_{1}, \ldots, X_{n}$, a utility function $u_{j}\left(x_{i j}\right)$ on the performance $x_{i j}$ of alternative $A_{i}$ under attribute $X_{j}$, a set of weights $w_{j}$ and the well-known functional form $u\left(A_{i}\right)=$ $\sum_{j=1}^{n} w_{j} u_{j}\left(x_{i j}\right), \forall i$. This represents the utility of each alternative $A_{i}, \forall i$. The incomplete information about input parameters has been incorporated into the decision-making process: (1) alternative performances under uncertainty and imprecision concerning utility function assessment, $u_{j}\left(x_{i j}\right) \in \boldsymbol{U}_{\boldsymbol{i j}}$; and (2) uncertainty about weights, which can be represented by: (a) ordinal relations, $\boldsymbol{W}=\left\{w: w_{1} \geq w_{2} \geq \cdots \geq w_{n}\right\} ;$ (b) value intervals, $\boldsymbol{W}=\left\{w: w_{j} \in\left[w_{j}^{L}, w_{j}^{U}\right]\right.$, $\forall j\} ;$ (c) intervals for weight ratios, $W=\left\{w: w_{j} / w_{k} \in\left[w_{j k}^{L}, w_{j k}^{U}\right]\right\}$; (d) linear inequality constraints for weights, $W=\{A W \leq c\}$; (e) nonlinear inequality constraints for weights, $\boldsymbol{W}=\{g(w) \leq 0\}$; (f) independent normal distributions, $W=\left\{w: w_{j} \sim N\left(\mu_{j}, \sigma_{j}^{2}\right), \forall j\right\}$; (f) fuzzy numbers, $\boldsymbol{W}=\left\{\widetilde{w}: \widetilde{w}_{j}, \forall j\right\}$, where $\widetilde{w}_{j}$ are triangular or trapezoidal fuzzy numbers.

In this section we propose a method based on the concept of dominance. Given two alternatives $A_{k}$ and $A_{l}$, alternative $A_{k}$ dominates $A_{l}$ if $D_{k l} \geq 0, D_{k l}$ being the optimum value of the optimization problem:

$$
D_{k l}=\min _{\substack{w \in W \\ u_{j}\left(x_{k j}\right) \in U_{k j}, \forall j \\ u_{j}\left(x_{l j}\right) \in U_{l j}, \forall j}}\left\{u\left(A_{k}\right)-u\left(A_{l}\right)=\sum_{j=1}^{n} w_{j}\left(u_{j}\left(x_{k j}\right)-u_{j}\left(x_{l j}\right)\right)\right\}
$$


The method is implemented in the following 4 steps.

1. Compute $D_{k l}$ for alternatives $A_{k}$ and $A_{l} \quad(\forall k, l)$ following (2) and the intervals $\boldsymbol{I}_{\boldsymbol{k} \boldsymbol{l}}=\left[D_{\boldsymbol{k} \boldsymbol{l}},-D_{\boldsymbol{l} \boldsymbol{k}}\right]$, if uncertainty about weights can be represented by style a), b), c), d) or e), see Section 2 .

2. Compute proportions $P_{k l}$ as follows:

a. If uncertainty about weights is represented by style a), b), c), d) or e), see Section 2, then

$$
P_{k l}=\left\{\begin{array}{cl}
d\left(\boldsymbol{I}_{k l}, 0\right), & \text { if } D_{k l} \geq 0 \\
\frac{-D_{l k}+D_{k l}}{-D_{l k}-D_{k l}} d\left(\boldsymbol{I}_{k l}, 0\right), & \text { if } D_{k l}<0 \text { and }-D_{l k}>0 \\
-d\left(\boldsymbol{I}_{k l}, 0\right), & \text { if }-D_{l k} \leq 0
\end{array}\right.
$$

b. If uncertainty about weights is represented by style f), then

$$
P_{k l}=\left(\int_{0}^{\infty} f_{k l}(x) d x\right) d\left(\boldsymbol{I}_{k l}, 0\right)-\left(1-\int_{0}^{\infty} f_{k l}(x) d x\right) d\left(\boldsymbol{I}_{k l}, 0\right)
$$

where $\mathrm{f}_{\mathrm{kl}}(\mathrm{x})$ is the density function of the variable Dkl and $\mathrm{I}_{\mathrm{kl}}=$ $[\mu-2 \sigma, \mu+2 \sigma]$;

a. If uncertainty about weights is represented by style $g$ ), then

$$
P_{k l}=\frac{\int_{0}^{\infty} f_{k l}(x) d x}{\int_{-\infty}^{\infty} f_{k l}(x) d x} d\left(D_{\boldsymbol{k} l}, 0\right)-\frac{\int_{-\infty}^{0} f_{k l}(x) d x}{\int_{-\infty}^{\infty} f_{k l}(x) d x} d\left(D_{k l}, 0\right)
$$

where $\mathrm{f}_{\mathrm{kl}}(\mathrm{x})$ is the membership function of the fuzzy number $D_{k l}$. Here $\mathrm{d}(\cdot, \cdot)$ is a distance measure ${ }^{8}$.

3. Compute a preference intensity measure for each alternative $A_{k}$ : $\mathrm{P}_{\mathrm{k}}=\sum_{\mathrm{l}=1}^{\mathrm{m}} \mathrm{P}_{\mathrm{kl}}$.

4. Rank alternatives according to the $P_{k}$ values, where the best (rank 1) is the alternative with greatest $P_{k}$ and the worst is the alternative with the least $P_{k}$.

\section{Simulation Study}

Let us compare the proposed method (NPIM) with the Mateos et al's ${ }^{5,6}(P I M)$, Ahn and Park's ${ }^{1}(A P), S M A A^{3}$ and $S M A A-2^{4}$ methods.

We propose to carry out a simulation study of the above methods to analyze their performance. The process would be as follows: (1) Randomly generate component utilities for each alternative in each attribute from a uniform distribution in $(0,1)$, leading to an $m \times n$ matrix. Remove dominated alternatives; (2) Generate attribute weights. Note that these weights are the TRUE weights, and the derived ranking of alternatives will be denoted as the TRUE ranking. The resulting weights will sum 1 and be uniformly distributed in the weight 
space; (3) To derive the corresponding weight intervals, add and subtract the same quantity to precise values, leading to the lower and upper endpoints of the weight intervals. We used the quantities, $q$, of $0.05,0.1,0.15,0.2$ and 0.25 that represent $10 \%, 20 \%, 30 \%, 40 \%$ and $50 \%$ imprecision, respectively. In other words, $\left[w_{i}{ }^{L}, w_{i}{ }^{U}\right]=\left[w_{i}{ }^{T}-q, w_{i}{ }^{T}+q\right]$. If $w_{i}{ }^{T}-q<0$, then $w_{i}{ }^{T}-q=0$, and if $w_{i}{ }^{T}+q>1$, then $w_{i}{ }^{T}+q=1$ is considered. Throughout the simulation process weights will be randomly generated from these weight intervals, $\left[w_{i}{ }^{T}-q, w_{i}{ }^{T}+q\right]$; (4) Compute the ranking of alternatives for each method according to their procedures and compare with the TRUE ranking, computed in step 2. We used two measures of efficacy, hit ratio and rank-order correlation'. The hit ratio is the proportion of all cases in which the method selects the same best alternative as in the TRUE ranking. Rank-order correlation represents how similar the overall structures ranking alternatives are in the TRUE ranking and in the ranking derived from the method. It is calculated using Kendall's $\tau$.

Four different levels of alternatives ( $m=3,5,7,10)$ and five different levels of attributes $(n=3,5,7,10,15)$ were considered in order to validate the results output. Also, 20,000 trials were performed for each of the 20 design elements (alternatives $\times$ attributes).

Table 1. Average hit ratios.

\begin{tabular}{crccccc}
\hline Alt. & Atrib. & NPIM & PIM & AP & SMAA & SMAA-2 \\
\hline 3 & 3 & 0.9937 & 0.9923 & 0.9813 & 0.9962 & 0.9962 \\
& 5 & 0.9878 & 0.9856 & 0.9744 & 0.9900 & 0.9901 \\
& 7 & 0.9778 & 0.9765 & 0.9610 & 0.9820 & 0.9824 \\
& 10 & 0.9652 & 0.9596 & 0.9459 & 0.9697 & 0.9698 \\
& 15 & 0.9300 & 0.9248 & 0.9043 & 0.9469 & 0.9470 \\
5 & 3 & 0.9902 & 0.9880 & 0.9697 & 0.9947 & 0.9951 \\
& 5 & 0.9815 & 0.9773 & 0.9540 & 0.9856 & 0.9857 \\
& 7 & 0.9709 & 0.9645 & 0.9404 & 0.9743 & 0.9752 \\
& 10 & 0.9504 & 0.9404 & 0.9163 & 0.9600 & 0.9606 \\
& 15 & 0.9110 & 0.90085 & 0.8701 & 0.9271 & 0.9273 \\
7 & 3 & 0.9906 & 0.9867 & 0.9667 & 0.9935 & 0.9935 \\
& 5 & 0.9768 & 0.9711 & 0.9446 & 0.9814 & 0.9818 \\
& 7 & 0.9657 & 0.9549 & 0.9249 & 0.9727 & 0.9732 \\
& 10 & 0.9438 & 0.9311 & 0.89985 & 0.9509 & 0.9512 \\
& 15 & 0.9025 & 0.8853 & 0.8455 & 0.9081 & 0.9087 \\
& 3 & 0.9895 & 0.9826 & 0.9603 & 0.9924 & 0.9926 \\
& 5 & 0.9753 & 0.9658 & 0.9317 & 0.9790 & 0.9798 \\
& 5 & 0.9602 & 0.9431 & 0.9079 & 0.9620 & 0.9631 \\
& 7 & 0.9379 & 0.9191 & 0.8807 & 0.9397 & 0.9406 \\
& 10 & 0.8944 & 0.8742 & 0.8326 & 0.8921 & 0.8925 \\
\hline
\end{tabular}


Table 1 exhibits the average hit ratio for each of the 20 design elements when the interval length is 0.1 , i.e., the average values of 20,000 trials.

If we consider the average rank-order correlation or other levels of imprecision, the results are very similar.

We can conclude that: (1) The new method and SMAA-2 are similar, and the difference between them is negligible; (2) The new method is easier to apply than SMAA-2 because the multidimensional integrals generally have to be computed using numerical techniques in SMAA-2; (3) The new method can be applied when uncertainty about weights is represented by fuzzy numbers, whereas SMAA-2 is not applicable in these cases; (4) All methods return a worse hit ratio when imprecision increases, as is to be expected.

\section{Conclusions}

In this paper we consider a new method to ranking alternatives when there is imprecision in the DM's preferences. This method is based on dominance measures. This method has the following advantages. First, it is applicable when the uncertainty about the weights of the additive multiattribute utility function can be represented by ordinal relations, value intervals, intervals for weight ratios, linear inequality constraints for weights, independent normal distributions or fuzzy numbers. Second, the method is one of the best considering the proportion of all cases in which the method selects the same best alternative or the rank-order correlation, as two measures of the goodness of the methods. Third, the method operations are very straightforward. The most complicated operation is the optimization problem, whereas integrals have to be solved to compute volumes to apply the SMAA or SMAA-2 methods. Numerical techniques are generally required to compute these integrals. Monte Carlo simulation was used to apply SMAA and SMAA-2.

A future research line is to analyze new preference intensity measures that consider the centroid values for the imprecision. 


\section{References}

1. B.S. Ahn and K.S. Park, Comparing Methods for Multiattribute Decision Making with Ordinal Weights, Comp. Oper. Res., 35, 1660-1670 (2008).

2. R.L. Keeney and H. Raiffa, Decision with Multiple Objectives: Preferences and Value Tradeoffs, Wiley, New York (1976).

3. R. Lahdelma, J. Hokkanen and P. Salminen, SMAA - Stochastic Multiobjective Acceptability Analysis, Eur. Oper. Res. 106, 1, 137-143, (1998).

4. R. Lahedelma and P. Salminen, SMAA-2: Stochastic Multicriteria Acceptability Analysis for Group Decision Making, Oper. Res., 49, 444454 (2001).

5. A. Mateos, A. Jiménez and J.F. Blanco, Ranking Methods Based on Dominance Measures Accounting for Imprecision, In: F. Rossi and A. Tsoukis (Eds.), ADT 2009, LNAI 5783, Springer-Verlag, 328-339 (2009).

6. A. Mateos A, A. Jiménez and J.F. Blanco, Two MCDM Ranking Methods Based on Preference Intensity Measures: Computational Study, Group Dec. and Negot., (in revision), 2012.

7. P. Sarabando and L.C. Dias, Simple Procedures of Choice in Multicriteria Problems without Precise Information about the Alternatives Values, Comp. Oper. Res., 37,12, 2239-2247 (2010).

8. L. Tran and L. Duckstein, Comparison of Fuzzy Numbers Using a Fuzzy Distance Measure, Fuzzy Sets and Systems 130, 331-341 (2002).

9. M. Weber, Decision Making with Incomplete Information, Eur. $J$. of Oper. Res., 28, 44-57 (1987). 